\title{
Nucleus-nucleus cross-sections and long-range correlations with a local supercritical pomeron
}

\author{
M.A.Braun \\ Dep. of High Energy physics, University of S.Petersburg, \\ 198504 S.Petersburg, Russia and \\ Dep. of Particle Physics, University of Santiago de Compostela, \\ 15782, Santiago de Compostela, Spain
}

October 27, 2018

\begin{abstract}
.
Nucleus-nucleus scattering is studied in the local Reggeon Field Theory in the quasi-classical approximation with non-eikonal boundary conditions corresponding to the Glauber picture at low energies. Comparison with the commonly used eikonal boundary conditions shows that the new conditions make both the action and nucleus-nucleus total cross-sections lower by $3 \div 5 \%$. They also substantially change the behaviour of the solutions of the equations of motion at low energies. Using expressions for the double inclusive cross-sections derived earlier in the Reggeon Field Theory [4] long-range rapidity correlations are calculated for the RHIC and LHC energies.
\end{abstract}

\section{Introduction}

Recent and forthcoming collider experiments attract much attention to the description of nucleusnucleus collisions. The corresponding amplitudes are very complicated already in the pure Glauber approach and become still more difficult to calculate in the pomeron approach with the triple pomeron interaction. In a recent publication the nucleus-nucleus amplitude was calculated within the tree approximation to the effective field theory of interacting perturbative QCD (BFKL) pomerons [1] taking into account non-eikonal boundary conditions, which are required to ensure the correct structure of the amplitude at low energies. It was found that in contrast to the situation with the eikonal boundary conditions, a whole set of different solutions follow from the relevant classical equations of motion. Both the mathematical reason and physical significance of this phenomenon are unclear. Is the higly non-linear form of the boundary conditions or rather the interplay between them and the structure of the BFKL pomeron responsible? A possible way to study this problem is to turn to a much simpler case of the old local pomeron described by the Local Reggeon Field Theory (LRFT) with the triple pomeron interaction, studied long ago with eikonal boundary conditions analytically in a series of elegant papers (see 2] and references therein). Apart from the pure theoretical interest, such a study may have a direct relation to the experimental situation, to the extent in which soft high-energy collisions can be described in the framework of LRFT.

Our results indicate that introduction of the improved non-eikonal boundary conditions into LRFT does not lead to dramatic changes which are observed in the QCD perturbative approach. True, it lowers the value of the action and also of the resulting cross-sections, as in the latter approach. However this effect is not at all large and, most significant, solutions of the classical equation do not infinitely multiply. So it is the interplay of the boundary conditions and inner 
structure of the pomeron, which leads to appearance of a family of solutions their number growing with rapidity. In fact behaviour of the solutions with rapidity is opposite: with its growth the number of solutions becomes smaller. From the experimental point of view, the predicted nucleus-nucleus cross-sections are much smaller than in the optical approximation, in accordance with the conclusions in [2]. The correction introduced by the non-eikonal boundary conditions is however relatively small.

The found cross-sections allow us to study the long-range rapidity correlations in particle production in nucleus-nucleus collisions, which have recently attracted much attention. In 3] qualitative predictions for the correlation coefficient $\sigma_{F B}$ of the forward-backward correlations have been presented in the framework of the the Color Glass Condensate model. It was argued that this coefficient should fall with the rapidity distance $\Delta y$ between the two windows and with the impact impact parameter and grow with the overall energy. However no concrete numerical values were obtained. In this paper we remind that in the framework of LRFT fully quantitative predictions for the correlations can be obtained. Of course this formalism does not allow to study the $p_{t}$ dependence of the inclusive spectra, which are to be treated either in the framework of the QCD (for the hard part of the spectra) or in models specially oriented to the soft part of the spectrum, such as colour string models. However for the long range correlations in rapidity of the spectra integrated over the transverse momenta LRFT seems to be fully adequate. So much the more that the highly non-trivial expression for the double inclusive cross-section in nucleus-nucleus collisions, necessary for the correlations, has up to the present been derived only in LRFT [4].

In 44 also the asymptotic expressions for the single and double inclusive cross-sections were found. However they refer to the case when all rapidity intervals, including the rapidity distance $\Delta y$, are very large, which does not correspond to the experimental setup. So in this paper, without introducing substantial new ingredients, we calculate the correlation coefficient $\sigma_{F B}$ from the old formuals and the found cross-sections for the present and future experimental situations.

The supercritical pomeron LRFT contains essentially three paramenters: the pomeron intercept $\alpha(0)=1+\mu$, its slope $\alpha^{\prime}(0)$ and three-pomeron coupling $\lambda$. The first and the last are more or less known. As for the slope, we take it equal to zero, since, on the one hand, all that is definitely known about it is that it is small and, on the other hand, its influence in heavy nuclear collisions should be expected only at energies much higher than the present and forthcoming ones. Last but not least, the problem with $\alpha^{\prime}(0)=0$ drastically simplifies and in fact allows to obtain analytical solutions for the inclusive spectra at a given point in the nuclear overlap area.

In fact the LRFT does not allow to calculate the correlation coefficient without any additional parameters. The contribution from the short-range correlations corresponding to the simulteneous production of the two observed particles from the pomeron is to be added to the rest part with an unknown weight $C$. This weight is the only new parameter which we introduce. Its good quality is that it is universal: it should not depend on any of the external variables coming from the experimental setup: $\Delta y$, overall rapidity $Y$, impact parametr $B$ atomic numbers of the collising nuclei etc. Its value can be inferred by comparison to the values of $\sigma_{F B}$ measured at RHIC for $\mathrm{Au}-\mathrm{Au}$ collisions.

Our results confirm the overall behaviour of ther correlation coefficient found in [3]: it grows with the overall energy and falls with $\Delta y$ and $B$.

The paper is organized as follows. In the next section we briefly recapitulate the basics of the LRFT. Section 3 is devoted to discussion of the boundary conditions for the equations of motion for the pomeronic fields. In Section 3. we present our numerical results for the total nucleus-nucleus cross-sections. In Section 4. they are used for calculation of the correlation coefficieint of the long-range rapidity correlations. Here also the necessary formulas for the single and double inclusive cross-sections are reproduced following 4]. The last section draws 
some conclusions

\subsection{Action of the LRFT and classical equations of motion}

The $S$-matrix for nucleus-nucleus collisions at impact parameter $B$ and rapidity $Y$ in the supercritical local pomeron model with a three-pomeron interaction is given by

$$
S(Y, B)=e^{-A_{\min }},
$$

where $A$ is the classical action

$$
A=\int d y d^{2} b \mathcal{L}
$$

in the presence of external sources, which only act at rapidities of the participant nuclei $y=0$ and $y=Y$. The Lagrangian is given by the sum of three terms

$$
\mathcal{L}=\mathcal{L}_{0}+\mathcal{L}_{I}+\mathcal{L}_{E}
$$

which represent free, interaction and external parts. The first two are given by

$$
\begin{gathered}
\mathcal{L}_{0}=\frac{1}{2}\left(\phi^{\dagger} \dot{\phi}-\dot{\phi}^{\dagger} \phi\right)+\mu \phi^{\dagger} \phi-\alpha^{\prime} \nabla \phi^{\dagger} \nabla \phi, \\
\mathcal{L}_{I}=-\lambda \phi^{\dagger} \phi\left(\phi^{\dagger}+\phi\right) .
\end{gathered}
$$

The form of the external part $\mathcal{L}_{E}\left(\phi, \phi^{\dagger}\right)$ will be discussed in the next section. In these expressions $\phi(y, b)$ and $\phi^{\dagger}(y, b)$ are two real fields, which describe in- and out-going pomerons with the intercept $\alpha(0)=1+\mu$, slope $\alpha^{\prime}$ and three-pomeron coupling $-\lambda$. $\dot{\phi}$ denotes the derivative in $y$, $\nabla$ refers to $b$. Due to smallness of the slope, following [2] we neglect the kinetic energy of the pomeron and study the simple case $\alpha^{\prime}=0$.

The classical equations of motion are then (at point $(y, b)$ )

$$
0=\delta A / \delta \phi^{\dagger}=\dot{\phi}+\mu \phi-\lambda \phi^{2}-2 \lambda \phi^{\dagger} \phi
$$

and

$$
0=\delta A / \delta \phi=-\dot{\phi}^{\dagger}+\mu \phi^{\dagger}-\lambda \phi^{\dagger^{2}}-2 \lambda \phi^{\dagger} \phi
$$

with the boundary conditions

$$
\phi(0, b) \delta(y)=d \mathcal{L}_{E} / d \phi^{\dagger}(y, b), \quad \phi^{\dagger}(Y, b) \delta(y-Y)=d \mathcal{L}_{E} / d \phi(y, b),
$$

which follow from the conditions

$$
\phi(y, b)=0 \text { at } y<0, \phi^{\dagger}(y, b)=0 \text { at } y>Y .
$$

Using the equations of motion one can find that parts of the classical action satisfy the relation

$$
A_{0}=-\frac{3}{2} A_{I}+\frac{1}{2} \int d^{2} b\left(\phi^{\dagger}(0, b) \phi(0, b)+\phi^{\dagger}(Y, b) \phi(Y, b)\right),
$$

which allows to exclude one part (say $A_{0}$ ) and write the classical action as

$$
A=-\frac{1}{2} A_{I}+A_{E}+\frac{1}{2} \int d^{2} b\left(\phi^{\dagger}(0, b) \phi(0, b)+\phi^{\dagger}(Y, b) \phi(Y, b)\right) .
$$




\section{External sources}

Each nucleon from the participant nuclei may emit any number of pomerons, so that the source term for nucleons in nucleus $\mathrm{A}$ is

$$
A T_{A}(b) \delta(y)\left(e^{-g \phi^{\dagger}(0, b)}-1\right)
$$

and the source term for nucleons in nucleus B is

$$
B T_{B}(B-b) \delta(y-Y)\left(e^{-g \phi(Y, b)}-1\right) .
$$

Here $T_{A(B)}$ are the standard nuclear profile functions, normalized to unity, $g$ is the nucleonpomeron coupling. Accordingly $S$ matrix at a fixed overall impact parameter $B$ will be given by

$$
\begin{gathered}
S(Y, b)=\int D \phi D \phi^{\dagger} e^{-A_{0}-A_{I}} \sum_{n_{A}, n_{B}} C_{A}^{n_{A}} C_{B}^{n_{B}} \\
\int \prod_{i=1}^{n_{A}} \prod_{j=1}^{n_{B}} d^{2} b_{i} d^{2} b_{j}^{\prime} T_{A}\left(b_{i}\right) T_{B}\left(B-b_{j}^{\prime}\right)\left(e^{-g \phi^{\dagger}\left(0, b_{i}\right)}-1\right)\left(e^{-g \phi\left(Y, b_{j}^{\prime}\right)}-1\right),
\end{gathered}
$$

where actions $A_{0}$ and $A_{I}$ are the free and interaction part of the action introduced in the preceding section. Summations over $n_{A}$ and $n_{B}$ lead to the final formula

$$
S(Y, b)=\int D \phi D \phi^{\dagger} e^{-A_{0}-A_{I}-A_{E}},
$$

where the external action $A_{E}$ is

$$
A_{E}=-A \ln \int d^{2} b^{\prime} T_{A}\left(b^{\prime}\right) e^{-g \phi^{\dagger}\left(0, b^{\prime}\right)}-B \ln \int d^{2} b^{\prime} T_{B}\left(B-b^{\prime}\right) e^{-g \phi^{\dagger}\left(Y, b^{\prime}\right)} .
$$

This form of the external sources leads to the boundary conditions

$$
\begin{gathered}
\phi(0, b)=A g T_{A}(b) \frac{e^{-g \phi^{\dagger}(0, b)}}{\int d^{2} b^{\prime} T_{A}\left(b^{\prime}\right) e^{-g \phi^{\dagger}\left(0, b^{\prime}\right)}}, \\
\phi^{\dagger}(Y, b)=A g T_{B}(B-b) \frac{e^{-g \phi(Y, b)}}{\int d^{2} b^{\prime} T_{B}\left(B-b^{\prime}\right) e^{-g \phi\left(Y, b^{\prime}\right)}} .
\end{gathered}
$$

As one observes, these boundary conditions are not only non-linear but they also mix values of the fields at $y=0$ and $y=Y$. So they do not represent initial conditions for evolution of the fields in rapidity but rather relate initial and fully evolved values of the fields.

One also observes that the complexity of these non-eikonal boundary conditions comes exclusively from the non-trivial $B$ and $b$ dependence of the nuclear distributions $T_{A}(b)$ and $T_{B}(B-b)$. Indeed in the (fictitious) case of constant profile functions, for central collisions $B=0$, both $\phi$ and $\phi^{\dagger}$ are constant in the common nuclear area and zero outside. Then conditions (17) become equivalent to the standard eikonal boundary conditions

$$
\phi(0, b)=A g T_{A}(b), \quad \phi^{\dagger}(Y, b)=A g T_{B}(B-b) .
$$




\section{Solution}

\subsection{Technique}

As in our previous studies ( [5, 1]) we searched for a solution of the classical equations of motion with given boundary conditions by iterations. We applied three different procedures. Most generally, at the first step we constructed $\phi(y, b)$ by evolution in $y$ of Eq. (6) with $\phi^{\dagger}(y, b)=0$ starting from its initial condition at $y=0$ obtained from (17) with $\phi^{\dagger}(Y, b)=0$. At the next step we evolved $\phi^{\dagger}(y, b)$ back according to Eq. (7) with already found values of $\phi(y, b)$ starting from its value at $y=Y$ obtained from (17) with the found value of $\phi(Y, b)$. Then this procedure is repeated. At each step both the equation of motion and initial values for $\phi(y, b)$ are taken with $\phi^{\dagger}(y, b)$ found in the previous iteration and vice versa. This procedure allows to find solutions which for identical nuclei $(A=B)$ are not symmetric in the interchange of the projectile and target, that is do not satisfy the relation

$$
\phi(b, y)=\phi^{\dagger}(B-b, Y-y)
$$

To specifically find such symmetric solutions we changed the procedure either by evolving both $\phi$ and $\phi^{\dagger}$ simulatenously or simply substituting evolution of $\phi^{\dagger}(y, b)$ by directly expressing it according to (19). Finally we repeated our calculations for the eikonal boundary conditions (18).

\subsection{Results}

In accordance with the experimental data we have taken

$$
\mu=0.08, \quad g_{N}=8.4 / \sqrt{2} \mathrm{GeV}^{-1}, \quad \lambda=\frac{\sqrt{2}}{25} g_{N}=0.48 \mathrm{GeV}^{-1} .
$$

(see Appendix). For the nuclear profile functions we have taken those which are generated by the Woods-Saxon nuclear density. We considered collisions of identical nuclei with $A=64$ and 197.

Our first series of results refers to values of the classical action at $B=0$ related to the $S$ matrix for central collisions as

$$
S(Y, B=0)=e^{-A(Y, B=0)}
$$

In Figs. 1 and 2 we show these values obtained for $\mathrm{Au}-\mathrm{Au}$ collisons with the non-eikonal boundary conditions (17), eikonal boundary conditions (18) and in the optical approximation when the action is

$$
g^{2} A B \int d^{2} b T_{A}(b) T_{B}(B-b)
$$

As mentioned, in contrast to the case of BFKL pomerons [1], in this local theory and with our choice of the couplings the equations of motion with the non-eikonal boundary conditions generate a finite number of solutions, different at rapidities lower or larger than a certain critical one, which is numerically found to be $Y_{c} \simeq 6$. At $Y<Y_{c}$ one gets a pair of solutions, which are not symmetric under the interchange of projectile and target nuclei. At $Y>Y_{c}$ this pair merges into a single symmetric solution. In Fig. 3 we show values of the fully evolved fields $\phi$ and $\phi^{\dagger}$ at the center $(b=0)$. As one observes, in the rapidity range $0<Y<6$ their values are very different but become equal at larger $Y$.

Under the eikonal boundary eikonal conditions the situation repeats the one found in [7]. At rapidities lower than $\sim 49$ a unique symmetric solution is found. At higher rapidities from this symmetric solution splits an asymmetric one, which realizes the minimum of the action. Both 


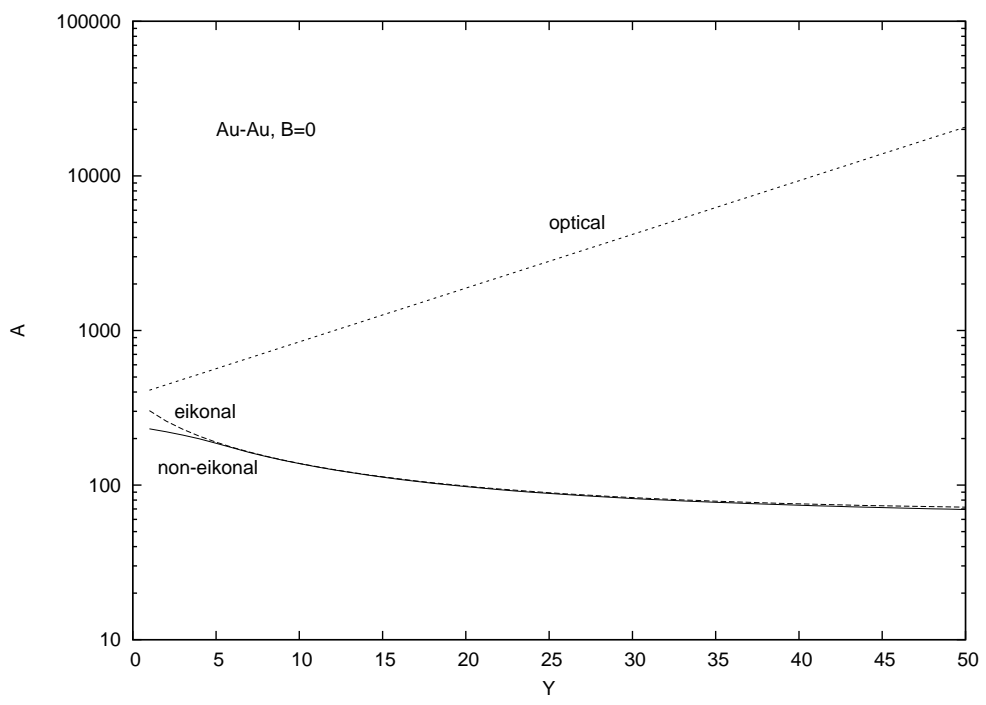

Figure 1: Minimal action obtained with the non-eikonal and eikonal boundary conditions as well as in the optical approximation as a function of rapidity for central $\mathrm{Au}-\mathrm{Au}$ collisions

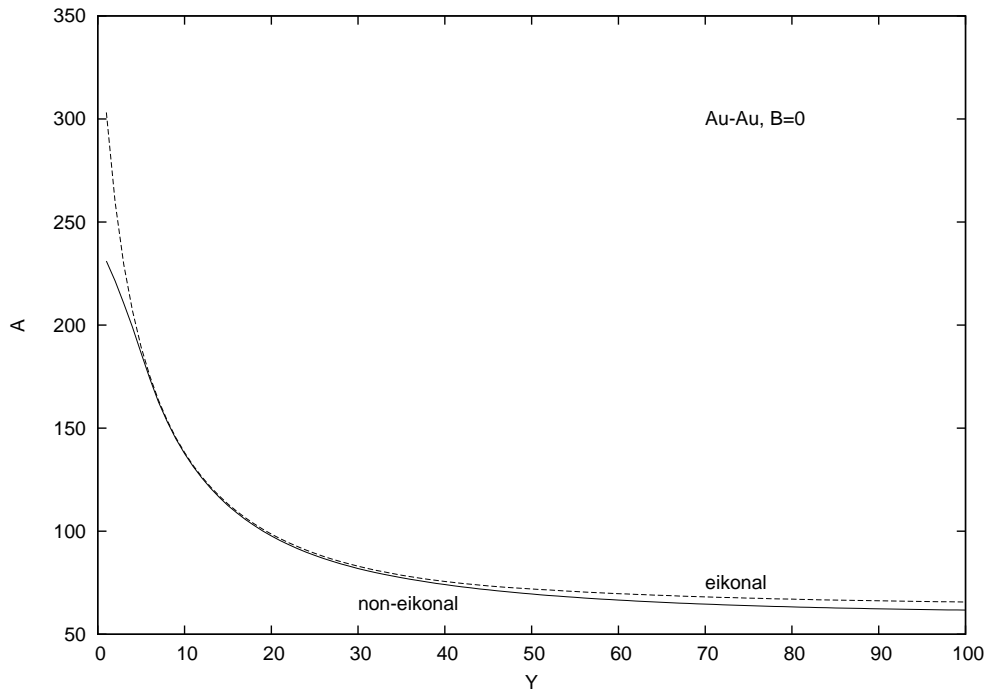

Figure 2: Minimal action obtained with the non-eikonal and eikonal boundary conditions in the extended interval of rapidities for central $\mathrm{Au}-\mathrm{Au}$ collisions. 


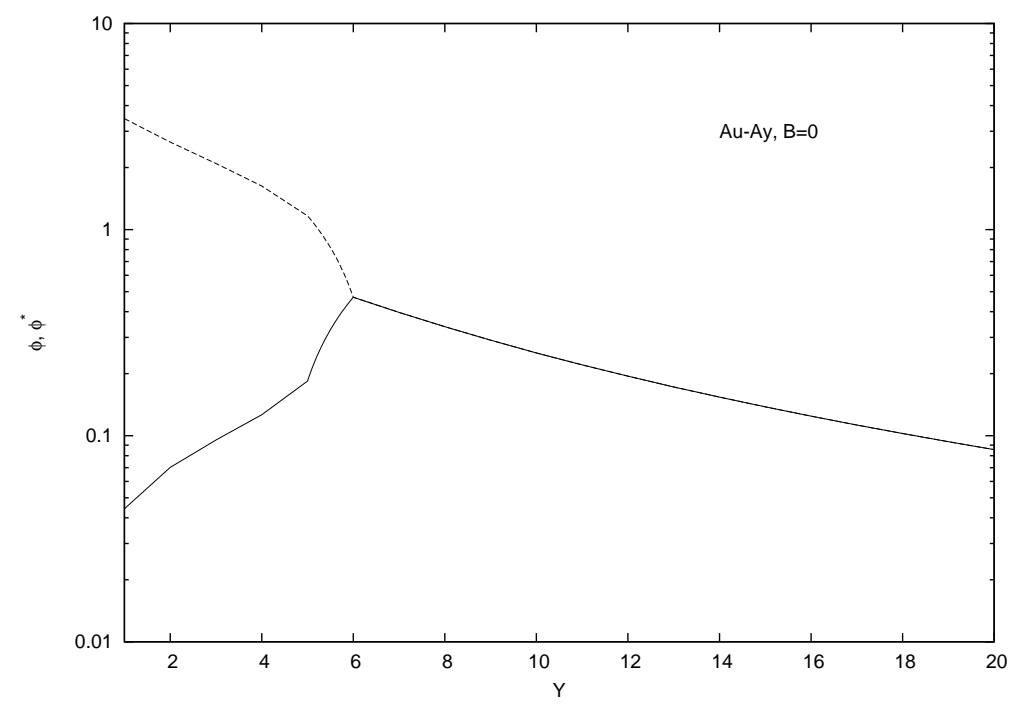

Figure 3: Values of the fully evolved fields $\phi$ and $\phi^{\dagger}$ at the center $(b=0)$. Below $Y=Y_{c}=6$ the upper-and lowermost curves correspond to the asymmetric pair of solutions.

solutions coexist up to $Y=82$ after which our procedures generate only a pair of asymmetric solutions.

As expected, with both types of boundary conditions the action is found to be much smaller than in the naive optical approximation. Moreover it slowly falls with rapidity. whereas the optical action naturally grows as the pomeron itself. Going to rapidities as high as 1000 we found that for both types of the boundary conditions the action freezes at values oly a few percent smaller than shown in Fig. 2 at $Y=100$. The difference introduced by the new non-eikonal boundary conditions is quite significant only at comparatively small rapidities $(\sim 30 \%)$ and in the high-energy limit goes down to $\sim 6.5 \%$.

At $B>0$ the situation with the non-eikonal boundary conditions is found to be the following. Until $B$ is greater than $B_{c}=8 \mathrm{fm}$ there exists a single symmetric solution, depending on the angle between $\mathbf{B}$ and $\mathbf{b}$ and realizing the minimum of the action, and a pair of asymmetric solutions As $B \rightarrow 0$ the symmetric solution passes into the pair of asymmetric solutions. At $B>B_{c}$ only a single symmetric solution exists. With the eikonal boundary conditions the problem is purely local, so that the behavior of solutions is qualitatively the same as for $B=0$.

In Fig. 4 we show values of the action for central $\mathrm{Cu}-\mathrm{Cu}$ collisions. Both with the non-eikonal and eikonal boundary conditions the action turns out to be proportional to $A^{2 / 3}$ as opposed to $A^{4 / 3}$ naively expected from the optical approximation. This dependence fully agrees with asymptotical estimates in [2]. The structure of the solutions repeats that for $\mathrm{Au}-\mathrm{Au}$ collisions with the same $Y_{c}=6$ and $B_{c}=3.5 \mathrm{fm}$.

The total inelastic nucleus-nucleus cross-sections are given by an integral over impact parameter $B$ :

$$
\sigma_{A B}^{i n}=\int d^{2} B\left(1-S^{2}(Y, B)\right)
$$

Here, in principle, we have to take into account a possible existence of several solutions of the classical equation of motion, which contribute to the $S$-matrix with different prefactors, determined by quantum corrections. In the purely local case we can use the result of [8]. For the eikonal boundary conditions in was found there that above the critical rapidity $Y_{c}$, when from a single symmetric solution splits a pair of asymmetric ones which give the minimum of the action, the $S$ matrix was given by the sum of contributions from the two asymmetric solutions minus the one from the symmetric solution. This guarantees continuity of the $S$-matrix at 


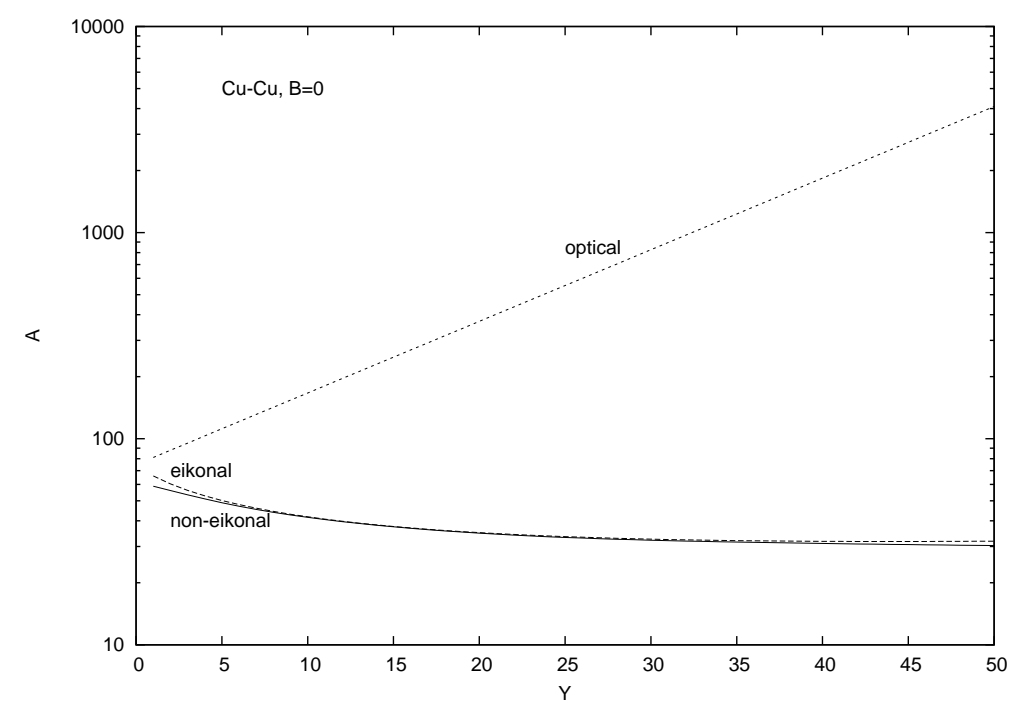

Figure 4: Same as Fig. [1 for central $\mathrm{Cu}-\mathrm{Cu}$ collisions

$Y=Y_{c}$. However this conclusion does not apply to the case of our non-eikonal boundary conditions, when both below and above the critical rapidity there may exist several solutions.

Fortunately for the calculation of the total cross-section this problem is of no relevance. In fact values for the action below the critical impact parameter $B_{c}$ are very large both for $\mathrm{Au}-\mathrm{Au}$ $(A>15$. $)$ and $\mathrm{Cu}-\mathrm{Cu}(A>10$.) collisions. So the contribution to the $S$-matrix from these $B$ is quite small and can safely be neglected altogether. At $B>B_{c}$, on the other hand, the only contribution comes from the single symmetric solution, so that the problem of relative weights does not arise.

The cross-sections calculated in this way are shown in Fig. 5 and 6 for $\mathrm{Au}-\mathrm{Au}$ and $\mathrm{Cu}-\mathrm{Cu}$ collisions respectively As one observes, the cross-sections calculated with the corrected noneikonal boundary conditions, eikonal boundary conditions and in the optical approximation all rise with rapidities albeit with a different rate. This rise is obviously due to the contribution from the peripheral collisions when the action becomes small. Physically this corresponds to the growth of the effective nuclear radius with energy. In the naive optical approximation the crosssections grow linearly with $Y$. With both the eikonal and non- eikonal boundary conditions this rise is weaker. Absolute values of the cross-sections are correspondingly the largest in the optical approximation and smallest with the non-eikonal boundary conditions, the difference between them growing with rapidity.

\section{Inclusive cross-sections}

In the old LRFT the inclusive cross-sections are obtained by attaching to the pomeron propagator emission verteces depending on the transverse momenta of the emitted particles. They are external to the model and are to be extracted from the experimental data. We shall not attempt to find these verteces and thus the absolute values of the inclusive spectra. Our interest lies in rapidity correlations, for which only inclusive spectra integrated over tranversal momenta are important and moreover the integrated emission verteces cancel in the expression for the correlation coefficient. So the formulas which we present below refer to the inclusive cross-sections without emission verteces. These formulas were obtained in 4] and we write them in their original notation with a slight change in the overall normalization corresponding to the standard definition of the single and double inclusive cross-sections (see Appendix). We 


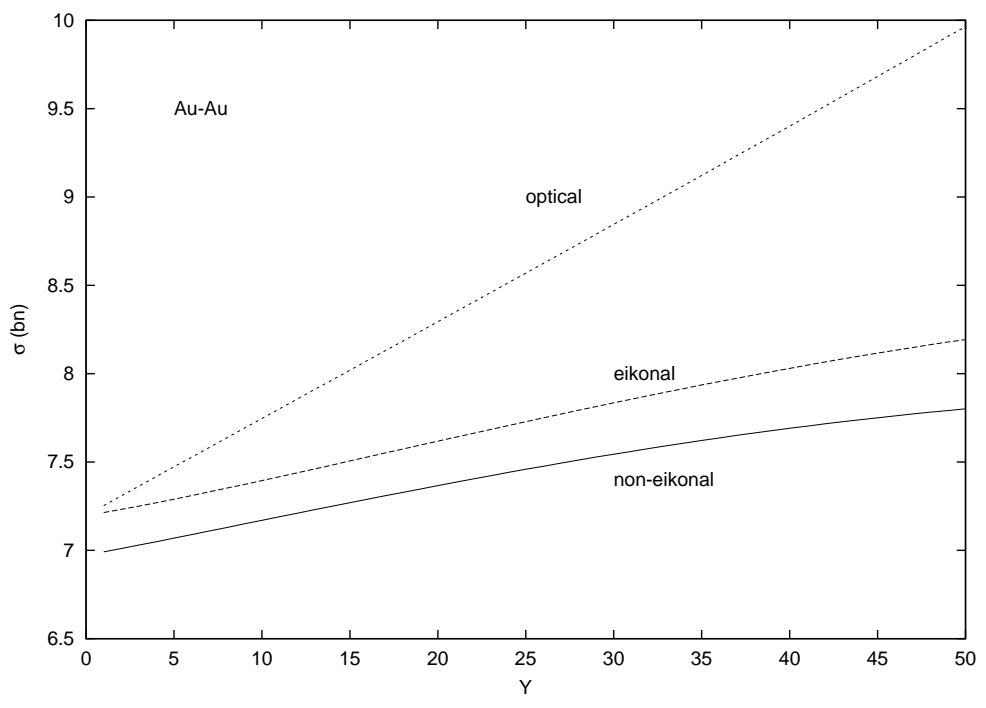

Figure 5: Total inelastic Au-Au cross-sections obtained with the non-eikonal and eikonal boundary conditions as well as in the optical approximation as a function of rapidity

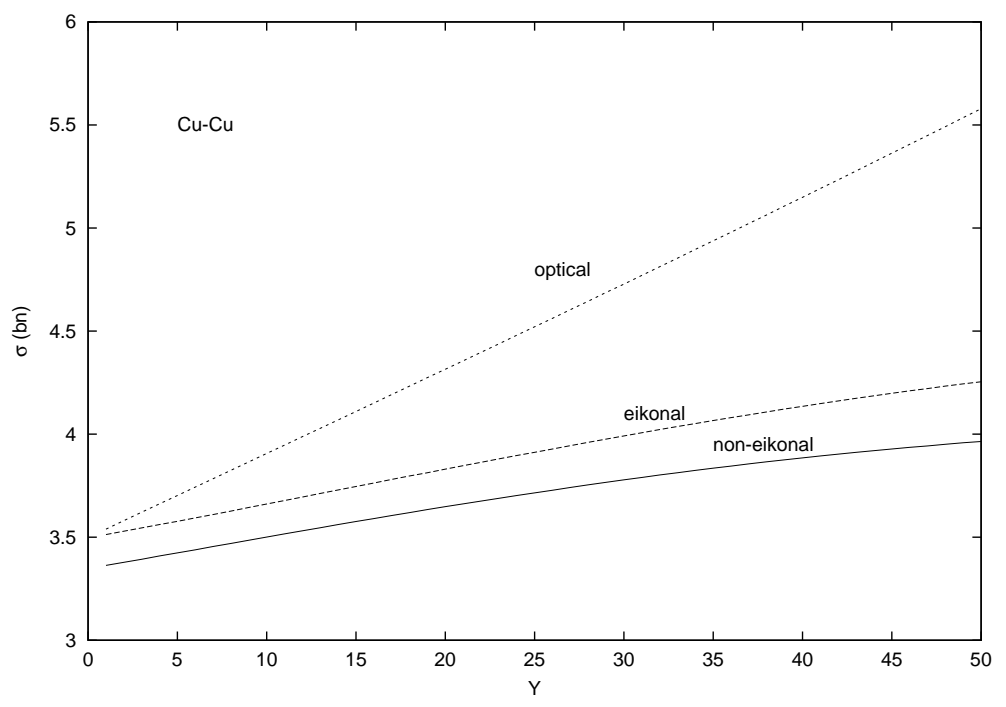

Figure 6: Total inelastic CU-Cu cross-sections obtained with the non-eikonal and eikonal boundary conditions as well as in the optical approximation as a function of rapidity 
stress that the approximation of zero zlope $\left(\alpha^{\prime}=0\right)$ is adopted. We also restrict ourselves to the simple case of collisions of identical nuclei at a fixed impact parameter $B$.

The single inclusive cross-section is then given by an integral over all transverse points $b$ inside the colliding nuclei

$$
I_{1}(Y, y, B)=\int d^{2} b I_{1}(Y, y, B, b),
$$

where $Y$ is the overall rapidity of the collision, $y$ is the rapidity of the observed particle and the inclusive cross-section $I_{1}(Y, y, B, b)$ at a given $b$ is a product of two sets of fan diagrams which start at the emission rapidity and go towards the colliding nuclei:

$$
I_{1}(Y, y, B, b) \equiv \frac{d \sigma}{d y d^{2} b}(Y, y, B, b)=2 \chi \tilde{\chi}
$$

Here $\chi$ is the sum of fan diagrams going from the emission point at rapidity $y$ to the target assumed to have rapidity zero:

$$
\chi=\frac{A g T_{A}(b) e^{\mu y}}{1+A g T_{A}(b) \frac{\lambda}{\mu}\left(e^{\mu y}-1\right)}
$$

and $\tilde{\chi}$ is the sum of fan diagrams going from the emission point to the projectile at rapidity $Y$ :

$$
\tilde{\chi}=\chi\left(y \rightarrow Y-y, T_{A}(b) \rightarrow T_{A}(B-b)\right) .
$$

The double inclusive cross-section for the emission at rapidity points $y_{1}$ and $y_{2}$ contains two terms. One is just a product of two single inclusive cross-sections

$$
I_{2}^{(1)}\left(Y, y_{1}, y_{2}, B\right)=I_{1}\left(Y, y_{1}, B\right) I_{1}\left(Y, y_{2}, B\right) .
$$

The other represents the triple pomeron and rescattering corrections. It is expressed via the pomeron Green functions $G\left(y_{1}, y_{2}\right)$ and $\tilde{G}\left(y_{1}, y_{2}\right)$ describing propagation of the pomeron in the external field created by the sums of fan diagrams $\chi$ and $\tilde{\chi}$ respectively:

$$
G\left(y_{1}, y_{2}\right)=\theta\left(y_{1}-y_{2}\right) e^{\mu\left(y_{1}-y_{2}\right)}\left[\frac{1+A g T_{A}(b) \frac{\lambda}{\mu}\left(e^{\mu y_{2}}-1\right)}{1+A g T_{A}(b) \frac{\lambda}{\mu}\left(e^{\mu y_{1}}-1\right)}\right]^{2}
$$

and

$$
\tilde{G}\left(y_{1}, y_{2}\right)=G\left(y_{1}, y_{2}\right)\left(y_{1} \rightarrow Y-y_{2}, y_{2} \rightarrow Y-y_{1}, T_{A}(b) \rightarrow T_{A}(B-b)\right) .
$$

The second part of the double inclusive is then

$$
\begin{gathered}
I_{2}^{(2)}\left(Y, y_{1}, y_{2}, B\right)=2 \tilde{\chi}\left(y_{1}\right) \chi\left(y_{2}\right)\left(G\left(y_{1}, y_{2}\right)+2 \lambda(G \chi \tilde{G})_{y_{1} y_{2}}\right) \\
+8 \lambda \tilde{\chi}\left(y_{1}\right) \tilde{\chi}\left(y_{2}\right)\left(G \chi G^{T}\right)_{y_{1} y_{2}}+\left(y_{1,(2)} \rightarrow Y-y_{1(2)}, T_{A}(b) \rightarrow T_{A}(B-b)\right) .
\end{gathered}
$$

The diagrammatic representation of these contributions can be found in [4]. We have to stress that at $y_{1}=y_{2}$ the two contributions to the double inclusive cross-section do not include emission of both particles at the same rapidity illustrated in Fig. 7. This contribution is important for correlations and should be added as

$$
\Delta I_{2}\left(Y, y_{1}, y_{1}\right)=C \tilde{\chi}\left(y_{1}\right) \chi\left(y_{1}\right)
$$

where the unknown coefficient $C$ represents the result of integration of the double emission vertex in Fig. 7 over transverse momenta of both particles divided by the square of integrated single emission verteces. 


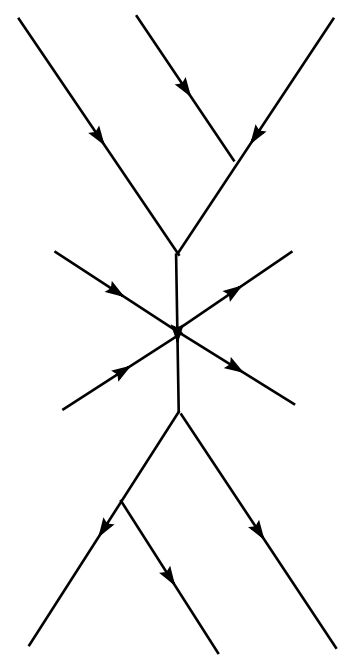

Figure 7: Diagrams with double emission at the same rapidity taken into account by (31)

Passing to the correlation coefficient we shall restrict ourselves to a situation when the forward window at $y_{1}$ and backward window at $y_{2}<y_{1}$ are symmetrically situated relative to the center:

$$
y_{1}=\frac{1}{2}(Y+\Delta y), \quad y_{2}=\frac{1}{2}(Y-\Delta y),
$$

with $\Delta y=y_{1}-y_{2}$ being the rapidity distance between the windows. We assume the width of both windows equal and small. Then at fixed impact parameter $B$ the correlation coefficient is given by

$$
\sigma_{F B}(Y, \Delta y, B)=\frac{\sigma^{i n}(B) I_{2}\left(Y, y_{1}, y_{2}, B\right)-I_{1}\left(Y, y_{1}, B\right) I_{1}\left(Y, y_{2}, B\right)}{\sigma^{i n}(B) I_{2}\left(Y, y_{1}, y_{1}, B\right)-I_{1}^{2}\left(Y, y_{1}, B\right)},
$$

where in the denominator $I_{2}\left(Y, y_{1}, y_{1}, B\right)$ includes the additional contribution (31). In this expression everything is known except coefficient $C$ in (31), which remains a parameter to be extracted from the comparison with the experimental data. This comparison with the RHIC data in [9] fixes $C=2.8$.

Once $C$ is fixed, the formalism allows us to find $\sigma_{F B}$ at all values of $Y, \Delta y$ and $B$. In Figs. 8-10 we show our results for $\mathrm{Au}-\mathrm{Au}$ collisions at values of $Y$ corresponding to RHIC and LHC energies and also to $Y=24$ to illustrate the general trend with the growth of energy. In Fig. 8 and 9 we show the $\Delta y$-dependence for central and peripheral collisions respectively. In Fig. 10 we show the $B$-dependence at $\Delta y=2$.

As we see, the correlation coefficient $\sigma_{F B}$ grows with $Y$ towards unity and at fixed $Y$ falls with $\Delta y$ with a slope which goes down with $Y$ and rises with $B$. As to the behaviour of $\sigma_{F B}$ in $B$, it practically remains constant until the collision becomes very peripheral, when it rapidly drops to zero. This behaviour generally agrees with experimental observations.

Calculations for $\mathrm{Cu}-\mathrm{Cu}$ collisions show that the correlation coefficient is practically independent of the atomic number except that the drop for peripheral collisions occurs at correspondingly lower values of $B$.

\section{Dicussion}

We have studied the nucleus-nucleus interaction in the framework of the old local Reggeon Field Theory with a supercritical pomeron having a vanishing slope $\alpha^{\prime}$. Loop contribution has been neglected. A new element in our treatment is introduction of new boundary conditions, 


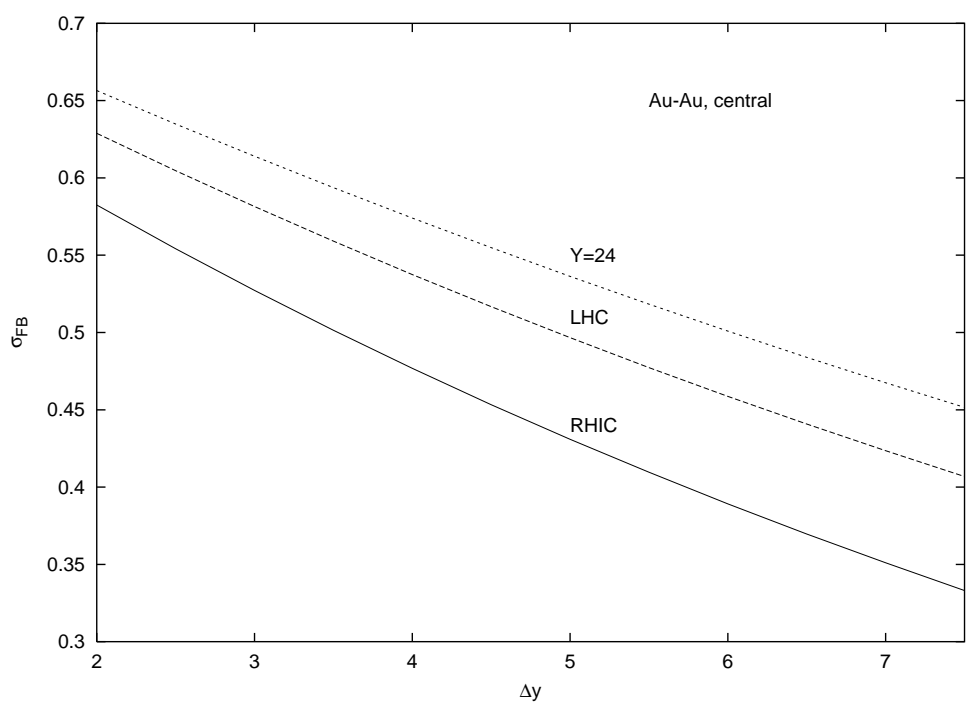

Figure 8: Correlation coefficient for central $\mathrm{Au}-\mathrm{Au}$ collisions as a function of distance between the forward and backward rapidity windows. Curves from bottom to top correspond to energies of RHIC, LHC and $Y=24$

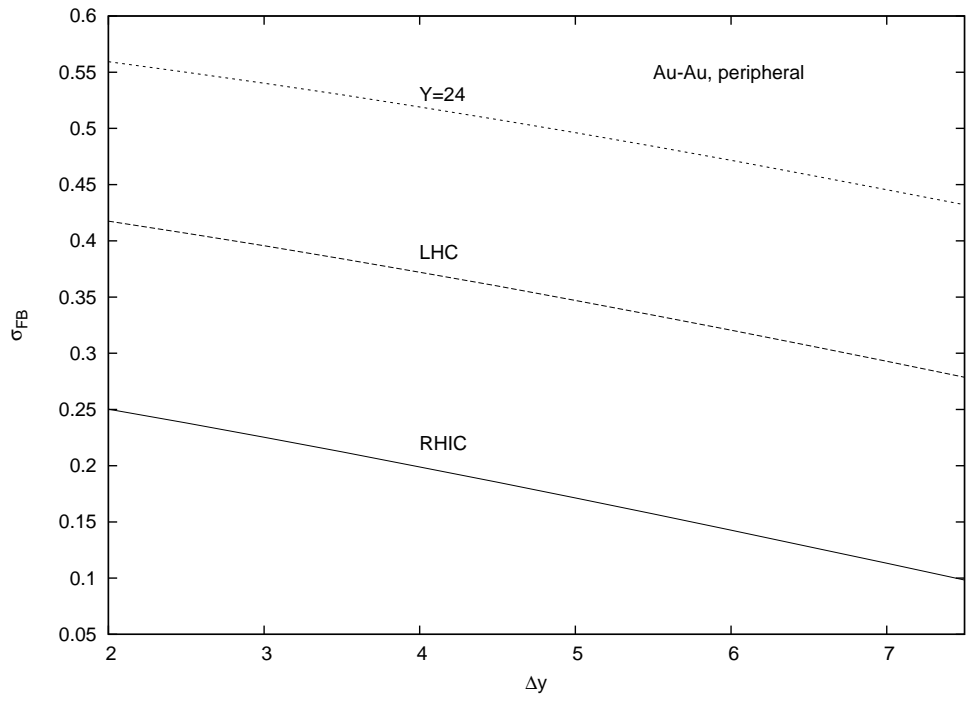

Figure 9: Same as in Fig. 8 for peripheral collisions 


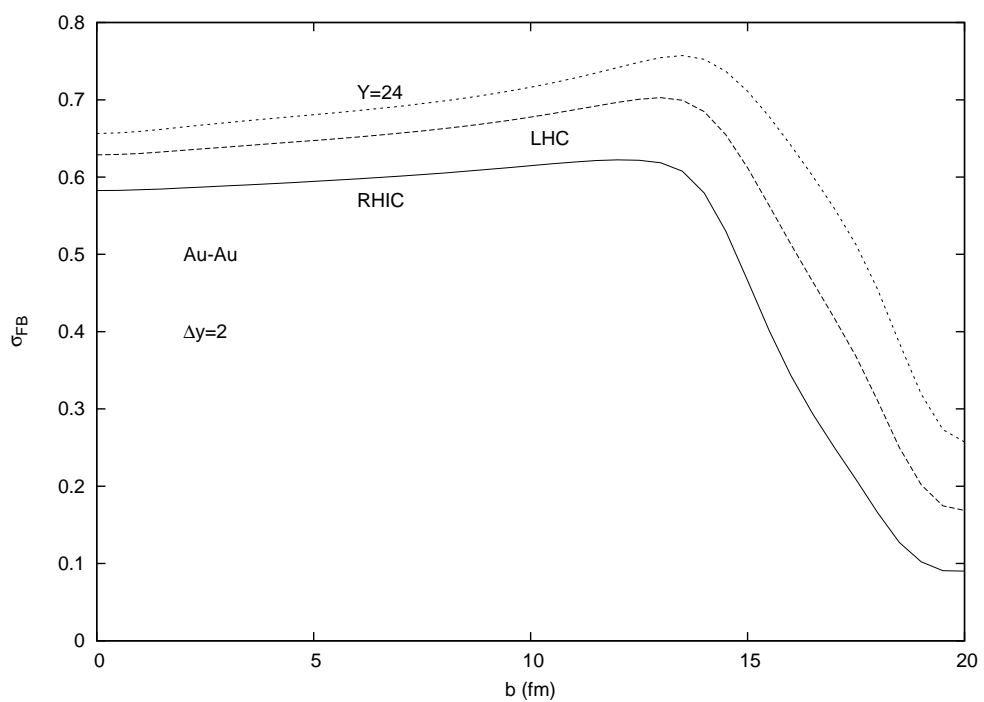

Figure 10: Correlation coefficient for $\mathrm{Au}-\mathrm{Au}$ collisions as a function impact parameter $B$ for $\Delta y=2$. Curves from bottom to top correspond to energies of RHIC, LHC and $Y=24$

which substitute more common eikonal boundary conditions to correspond to the standard Glauber expression at low rapidities. The new boundary conditions are very complicated from the mathematical point of view: they are non-local both in coordinates and rapidity.

Our found iterative solutions show that the actual change in the the action and cross-sections due to these new boundary conditions is not very large $(3 \div 5 \%$ for the cross-sections). However the structure of the solution is radically changed. Now the most complicated region is that of low rapidities, where there exist only a pair of asymmetric solutions and no symmetric one. This situation is reminiscent to that in the old studies of the pure Glauber approximation (without loops), when it was found that symmetric solutions exist only in the limited interval of atomic numbers 6].

The situation with the local pomeron is to be contrasted to that with the BFKL pomeron, where non-eikonal boundary conditions lower the action at $B=0$ significantly [1] and one can expect a corresponding drop in the total cross-sections.

The long-range rapidity correlations extracted from the single and double inclusive crosssections and the found total cross-sections behave with overall energy, centrality and rapidity distance in accordance with conjectures made in another approaches. Adjusting our only parameter to the experimental value of the correlation coefficient $\sigma_{F B}$ at RHIC energy, $B=0$, and $\Delta y=2$ we have predictions for $\sigma_{F B}$ at any values of energy, $B$ and $\Delta y$. Measurement of $\sigma_{F B}$ at these experimental conditions will shed new light on the validity of this simple local supercritical pomeron approach.

\section{Acknowledgements}

The author benefited from numerous constructive discussions with N.Armesto, S.Bondarenko, C.Pajares and Yu.Shabelsky. The author thanks MEC of Spain for financial support under the project FPA 2005-01963. This work was also supported by grants RNP 2.1.1.1112 and RFFI 06-02-16115a of Russia. 


\section{Appendix. Normalization of reggeon amplitudes}

To relate the amplitudes obtained within LRFT with the physical cross-sections it is sufficient to study the simple case $\lambda=0$ corresponding to the pure Glauber rescattering. At high energies the relativistic forward scattering amplitude $A$ is related to the total cross-section by the standard relation $\sigma^{t o t}=\operatorname{Im} A / s$. Passing to the LRFT theory, which lives in the purely transversal space, one has to integrate over longitudinal variables in each loop. This integration gives a factor $1 /(2 s)$ for each loop. The total coefficient for $n$ pomeron exchanges and thus $(n-1)$ integration loops is $g^{2 n} s^{n} \cdot(2 s)^{1-n}=2 s\left(g^{2} / 2\right)^{n}$. So factorization of the contribution into the product of exchanges generates factor $2 s$. The cross section obtained after division by $s$ aquires factor 2 . So the correct relation between the cross-section and the amplitude in LRFT at fixed $B$ is

$$
\sigma^{t o t}(B)=2 \operatorname{Im} A_{L R F T}(B)=2(1-S(B)) .
$$

The elastc and inelstic cross-sections are then

$$
\sigma^{e l}(B)=(1-S(B))^{2}, \quad \sigma^{i n}(B)=1-S^{2}(B) .
$$

With these relations in view one has to take into account that the standard (experimental) definitions of the coupling constants $g$ and $\lambda$ are taken from the cross-sections. This implies that if $g_{E}$ and $\lambda_{E}$ are the experimental values then the parameters $g$ and $\lambda$ in the LRFT Lagrangian are determined by the relations $g_{E}^{2}=2 g^{2}, \quad g_{e}^{3} \lambda_{E}=2 g^{3} \lambda$ from which it follows

$$
g=g_{E} / \sqrt{2}, \quad \lambda=\lambda_{E} \sqrt{2} .
$$

These definitions are used in (20).

\section{References}

[1] S.Bondarenko and M.A.Braun, arXiv:0708.3629.

[2] D.Amati, L.Caneshi and R.Jengo, Nucl. Phys. B 101 (1975) 397.

[3] N.Armesto, L.McLerran and C.Pajares, Nucl. Phys. A 781 (2007) 201.

[4] M.Ciafaloni, G.Marchesini, Nucl. Phys. B 109 (1976) 261.

[5] M.A.Braun, Eur. Phys. J C 33 (2004) 113.

[6] A.S.Pak, A.V.Tarasov, V.V.Uzhinsky, Ch.Tzeren, Yad. Fiz. 30 (1979) 102.

[7] S.Bondarenko and L.Motyka, hep-ph/0605185.

[8] M.Ciafaloni, Nucl. Phys. B 146 (1978) 427.

[9] B.K.Srivastava (STAR collab), arXiv:nucl-ex/0702054; T.J.Tarnowsky (STAR collab), arXiv:0711.1175 [nucl-ex]. 